\title{
Revisión de la Enfermedad de Addison en adultos.
}

\section{Review of Addison's Disease in adults.}

Omar Banegas Peña' Norma Banegas Peña ${ }^{2}$ Hilver Barillas Lamuño3 Alejandro José Corella Solano ${ }^{4}$

Jessica Riday Morúa ${ }^{5}$

1,2,3,4 y 5 Médico general. Trabajador independiente, San José Costa Rica.

Contacto: omarbanegas27@gmail.com

\section{RESUMEN}

La enfermedad de Addison es el término utilizado para referirse a la insuficiencia adrenal primaria. Esta condición es poco común y se caracteriza por un déficit crónico de glucocorticoides y/o mineralocorticoides. La principal causa es autoinmune, pero también puede haber etiologías infecciosas, neoplásicas, genéticas o iatrogénicas. Para su diagnóstico se requiere de una alta sospecha clínica. Su tratamiento se basa en la reposición farmacológica de los esteroides adrenales deficientes.

Palabras Clave: enfermedad de Addison, insuficiencia adrenal, cortisol, glucocorticoides, mineralocorticoides.

\section{ABSTRACT}

Addison's disease is the term used to refer to primary adrenal insufficiency. This condition is rare and is characterized by a chronic deficiency of glucocorticoids and / or mineralocorticoids. The main cause of this is autoimmune, but there can also be infectious, neoplasic, genetic, or iatrogenic etiologies. A high clinical suspicion is required for its diagnosis. Its treatment is based on the pharmacological replacement

Cómo citar:

Banegas Peña, O.,

Banegas Peña, N., Barillas Lamuño, H., Corella Solano, A.

J., \& Riday Morúa,

J. Revisión de la

Enfermedad de Addison en adultos.

Revista Ciencia Y

Salud Integrando

Conocimientos, 5(4),

Pág. 63-70. https://

doi.org/10.34192/

cienciaysalud.v5i4.310 of deficient adrenal steroids.

Keywords: Addison's disease, adrenal insufficiency, cortisol, glucocorticoids, mineralocorticoids..

Recibido: 28/abr/2021

Aceptado: 16/ago/2021

Publicado: 04/sep/2021 


\section{INTRODUCCIÓN}

En el año 1855, en la ciudad de Londres, el doctor Thomas Addison publica un reporte de 11 pacientes que mueren de "anemia, disminución de la actividad cardíaca, cambios de color en la piel, asociado a una enfermedad suprarrenal, definiendo este desorden como melasma suprarrenal". La causa de este daño suprarrenal se debía a tuberculosis, cáncer, hemorragia y fibrosis idiopática. En 1856, el doctor Trousseau utilizó el término "enfermedad bronce de Addison"; luego se le daría el nombre de enfermedad de Addison (1).

Se produce una insuficiencia adrenal primaria cuando la corteza suprarrenal es incapaz de producir glucocorticoides y/o mineralocorticoides para las necesidades corporales. Basándose en la fisiología de que estas hormonas, son reguladoras de la homeostasis hidroelectrolítica y del balance energético; es una enfermedad que puede comprometer la vida si se presenta de manera aguda, aunque se presenta de manera insidiosa en la mayoría de los casos $(1,2)$. Su etiología es principalmente autoinmune en países desarrollados. Los síntomas inespecíficos con los que cursa esta enfermedad provocan que sea difícil de reconocer y es frecuentemente infradiagnosticada (3). Su pronóstico ha mejorado de manera dramática con la disponibilidad farmacológica de esteroides suprarrenales sintéticos (2).

En este artículo se hace una revisión de la epidemiología, etiología, fisiopatología, clínica, diagnóstico y tratamiento de la enfermedad de Addison.

\section{MÉTODOS}

Para la realización de este artículo, se hizo una búsqueda en las bases de datos electrónicas PubMed, Elsevier y Uptodate con las frases "Enfermedad de Addison", "Insuficiencia Suprarrenal”, "Hipocortisolismo", "Síndrome Poliglandular Autoinmune" y "Adrenalitis". Se incluyeron revisiones de temas y publicaciones originales creadas entre el período 2016-2021. Se analizaron documentos en idiomas español e inglés. En total se recopilaron ?? artículos y 1 libro de texto para esta revisión.

\section{EPIDEMIOLOGÍA}

La insuficiencia adrenal primaria es una enfermedad muy rara. Sin embargo, con el avance del tiempo los casos aumentan. Este incremento en casos se ha debido a la poca información que se tenía sobre este padecimiento antes y a los avances en diagnóstico (4). Generalmente se manifiesta entre la segunda y cuarta década de vida, mayoritariamente en mujeres (4,5). Actualmente tiene una prevalencia de 2 en 10.000 personas y generalmente es más común en adultos que en niños $(6,7)$. La prevalencia puede estar afectada por factores como la variación genética, factores ambientales y diagnóstico correcto.

\section{FISIOLOGÍA}

Por definición, la insuficiencia adrenal es una condición clínica causada por falla en la síntesis de cortisol en la glándula suprarrenal (8). Para la síntesis de dicha hormona, se requiere de un adecuado eje hipotálamohipófisis-suprarrenal; en donde la hormona liberadora de hormona adrenocorticotropa ( $\mathrm{CRH}$ ) es liberada del hipotálamo ejerciendo su efecto sobre la adenohipófisis para que esta a su vez termine liberando la hormona adrenocorticotropa (ACTH) que llega a estimular la corteza de la glándula suprarrenal, específicamente en la zona fascicular, para la liberación de cortisol, el cual a su vez termina ejerciendo efecto de retroalimentación negativa sobre el eje (8).

El cortisol por su parte, siendo un compuesto hidrófilo, es soluble en plasma pero a su vez tiene alta capacidad lipofílica, otorgándole la posibilidad de unirse a receptores intracelulares de células diana, los cuales están localizados en casi todos los tejidos del cuerpo (9). Dicho complejo cortisol-receptor eventualmente se translocará al núcleo donde participará en el aumento o disminución de la expresión de múltiples genes (9); los efectos a nivel nuclear del cortisol terminarán ejerciendo diferentes efectos sobre fisiología corporal, 


\section{CIENCIA\&SALUD}

entre los cuales se pueden mencionar: elevar niveles plasmáticos de glucosa, aumento en la reabsorción de sodio, expandiendo el compartimento extracelular; esto a su vez, aumentando las cifras tensionales, actividad inmunosupresora y antiinflamatoria, efecto sobre metabolismo de proteínas y lípidos, afectación sobre el sistema nervioso central, efectos en metabolismo de calcio y hueso, entre otros (9).

Por otro lado, por medio del sistema renina-angiotensina-aldosterona, la corteza suprarrenal juega un papel importante, con la liberación de aldosterona de la zona glomerular en la homeostasis de sodio y mantenimiento de volemia. (9)

\section{PATOGÉNESIS}

La insuficiencia suprarrenal puede clasificarse de acuerdo con la localización anatómica en la cual ocurre el fallo para mantener un funcionamiento adecuado del eje que termina en una ausencia de cortisol. Insuficiencia adrenal primaria hace referencia a las alteraciones que ocurren a nivel de la glándula suprarrenal causada por diferentes etiologías, las cuales se mencionan posteriormente en esta revisión; insuficiencia adrenal secundaria ocurre cuando hay deficiencia en la secreción de ACTH debido a patología a nivel hipotálamo o en la glándula pituitaria; tomando también en consideración supresión de la liberación de ACTH debido a exposición excesiva a glucocorticoide exógeno. (8)

Con respecto de la insuficiencia suprarrenal primaria, previamente la causa más común de esta era debido a una infección por tuberculosis, actualmente se sabe que la principal causa es la insuficiencia suprarrenal autoinmune o Enfermedad de Addison (9).

La adrenalitis autoinmune o Enfermedad de Addison ocurre de forma aislada en un 30-40\% de los casos de insuficiencia adrenal primaria, sin embargo esta etiología también puede formar parte del Síndrome Poliglandular Autoinmune (8). El Síndrome Poliglandular Autoinmune a su vez presenta 2 tipos, el Tipo 1, el cual ocurre en $10 \%$ de los casos de insuficiencia adrenal primaria, es una enfermedad autosómica recesiva, la cual también se conoce como poliendocrinopatia autoinmune, candidiasis y distrofia ectodérmica o APECED por sus siglas en inglés (autoinmune polyendocrinopathy, candidiasis and ectodermal dystrophy) (8). Sin embargo, es más frecuente el Síndrome Poliglandular Autoinmune tipo 2 en estos individuos, presentándose en 50-60\% de los pacientes con insuficiencia adrenal primaria. (8) Dicho síndrome típicamente se presenta en la adultez y asocia además otras enfermedades autoinmunes como vitíligo, diabetes mellitus tipo 1 , patología tiroidea autoinmune, anemia perniciosa, enfermedad celiaca e insuficiencia gonadal primaria (8). Específicamente se requiere que el paciente padezca dos de las siguientes tres patologías para considerarlo como Síndrome Poliglandular Autoinmune tipo 2: Insuficiencia adrenal primaria, Diabetes mellitus tipo 1 y patología tiroidea autoinmune (10).

Con respecto al mecanismo inicial de la patogénesis, todavía se sabe poco respecto al inicio de la misma. Lo que es conocido de forma sistemática es que para que ocurra la autoinmunidad, se requiere de susceptibilidad genética y un evento precipitante (10). Infecciones virales y otras infecciones locales han sido consideradas entre los eventos precipitantes, ya que pueden llevar a una exposición de las células adrenales a diferentes productos microbianos y citoquinas, lo cual genera alteraciones en dichas células y afecta su relación con el sistema inmunológico (10). Específicamente, un virus como el herpes simplex virus 1, citomegalovirus y adenovirus poseen tropismo por las células de la corteza adrenal (10).

Durante la patogénesis de la enfermedad, la enzima 21a-hidroxilasa es el principal objetivo de los autoanticuerpos (10). Dicha afectación autoinmune es evidenciada de forma característica, entre los hallazgos histopatológicos de la Enfermedad de Addison, con infiltración linfocítica de la corteza adrenal asociando atrofia del parénquima sin afectar la médula adrenal (10). Sin embargo, la enfermedad podría permanecer silente y manifestarse hasta el momento en que ocurra la destrucción del 90\% de las células (10). Debido a la afectación de forma generalizada sobre la corteza suprarrenal en la insuficiencia adrenal primaria, la liberación de andrógenos y aldosterona también se ven comprometidas en esta patología. 


\section{CIENCIA\&SALUD}

Por otro lado, entre etiologías infecciosas de insuficiencia adrenal primaria, es la tuberculosis extrapulmonar, siendo la glándula suprarrenal uno de los sitios más frecuentemente afectados. Aunque dicha etiología infecciosa ya no se considera la principal causa de insuficiencia adrenal primaria en países desarrollados, todavía es causa importante de dicha patología en países en vías de desarrollo donde la tuberculosis es endémica (8).

La Hiperplasia Suprarrenal Congénita hace referencia a diferentes trastornos que afectan la esteroidogénesis a nivel de la glándula suprarrenal, secundaria a deficiencias enzimáticas, siendo la más afectada la 21a-hidroxilasa en más de $90 \%$ de los casos (8).

Ciertos medicamentos pueden generar insuficiencia adrenal de diferentes maneras. Fármacos como ketoconazol, fluconazol, itraconazol, etomidato inhiben las enzimas involucradas en la esteroidogenésis mitocondrial (8). Por otro lado, drogas como fenitoína o fenobarbital son inductores enzimáticos a nivel hepático, específicamente enzimas de CYP3A4, los cuales metabolizan el cortisol activo en 6ß-hidroxicortisol (8). Estudios recientes también han demostrado que ciertos medicamentos inhibidores de puntos de control inmunitario han sido relacionados con aumento en la incidencia de fenómenos autoinmunes (8).

Otras etiologías de insuficiencia adrenal primaria se mencionan en el anexo 1 así como etiologías de insuficiencia adrenal secundaria y terciaria.

\begin{tabular}{|c|l|}
\hline Etiología & \multicolumn{1}{|c|}{ Insuficiencia Suprarrenal Primaria } \\
\hline Autoinmune & Adrenalitis Autoinmune, Síndrome Poliglandular Autoinmune \\
\hline \multirow{2}{*}{ Infeccioso } & -Bacteriano: Tuberculosis \\
& -Viral: CMV, VIH \\
& -Fúngico: Histoplasmosis \\
\hline Vascular & Hemorragia adrenal, vasculitis, síndrome de Waterhouse- \\
& Friderichsen \\
\hline Infiltrativo & Hemocromatosis, Amiloidosis, Sarcoidosis \\
\hline Tumoral & Linfoma adrenal primario, metástasis \\
\hline \multirow{2}{*}{ Inducido por Drogas } & -Inhibición de síntesis de cortisol: Ketoconazol, \\
& fluconazol, etomidato \\
& -Inductores de CYP3A4: Fenitoína, Fenobarbital, \\
& Rifampicina \\
& -Inhibidores de puntos de control inmunitario \\
& -Litio \\
\hline Iatrogenia & -Adrenolíticos: Mitotano \\
\hline Congénito & Adrenalectomía Bilateral \\
\hline
\end{tabular}

Anexo 1. Etiologías de la Insuficiencia Suprarrenal Primaria (J).

Afectación Sistémica en la Insuficiencia Adrenal Primaria

Trastornos de Electrolitos y Fluidos:

-Hiponatremia: El déficit de cortisol puede generar hiponatremia por medio de diferentes mecanismos, entre los cuales se encuentran la disminución en la capacidad renal para excretar agua, generando hiponatremia dilusional, ausencia en el eje de retroalimentación negativa que ejerce el cortisol sobre la liberación de hormona antidiurética; en casos que se asocie deficiencia de aldosterona también 


\section{CIENCIA\&SALUD}

presenta disminución en la capacidad para reabsorción de sodio y agua a nivel de túbulo distal y colector (10).

-Hipotensión: Secundario a la deficiencia en la capacidad de reabsorción de sodio y agua ocurre una disminución en la volemia, afectando el compartimento extracelular, lo que disminuye la precarga, gasto cardíaco y como consecuencia, la presión arterial.

-Hiperpotasemia: La deficiencia de aldosterona compromete la bomba de sodio potasio dependiente de ATP a nivel renal, la cual es indispensable para mantener la función adecuada en la reabsorción de sodio y agua así como la eliminación de potasio (10).

-Hipercalcemia: Secundaria a la disminución en la filtración glomerular y por ende disminución en la eliminación renal de calcio, participando otros mecanismos como aumento en la salida del calcio al espacio extracelular (10).

\section{Trastornos Metabólicos:}

-Hipoglicemia: Tomando en consideración la función del cortisol en la gluconeogénesis hepática, una deficiencia del mismo cursará con hipoglicemia (10).

-Alteración Tiroidea: El cortisol atenúa la liberación de la hormona estimuladora de tiroides (TSH), cuyo efecto se pierde en la insuficiencia adrenal primaria llevando a un aumento ligero en los niveles de TSH asociando a su vez niveles normales o ligeramente disminuidos de hormona tiroidea como resultado de un Eutiroideo Enfermo, lo cual podría ser equívocamente confundido con Hipotiroidismo (10). El riesgo de iniciar reemplazo de hormona tiroidea en estos pacientes es que podría precipitar una crisis adrenal al aumentar el aclaramiento de cortisol, tasa metabólica y con esto el requerimiento de cortisol (10).

\section{Trastornos Dermatológicos:}

-Hiperpigmentación: Ante la deficiencia de cortisol, se pierde el eje de retroalimentación negativa ejercido sobre el eje hipotálamo-hipófisis-adrenal; esto llevará a un aumento tanto en ACTH así como la hormona estimuladora de melanocitos (MSH). La MSH estimulará la síntesis de melanina por parte de los melanocitos epidérmicos (10).

\section{CLÍNICA}

La insuficiencia adrenal primaria es subdiagnosticada debido a sus síntomas inespecíficos requiriendo de una alta sospecha clínica para diagnosticarlo $(8,11)$. Se ha descrito una alta incidencia de errores en el diagnóstico (68\%) confundiéndose más comúnmente con patologías psiquiátricas y gastrointestinales (8). Las manifestaciones clínicas varían desde una crisis adrenal aguda hasta una presentación crónica insidiosa (8).

Es importante recalcar que la crisis adrenal es una de las emergencias endocrinas más temidas, cuyo diagnóstico inicial es clínico y requiere de un manejo inmediato, sin demora a los resultados de laboratorio $(8,12)$. Ésta se desencadena usualmente en pacientes con insuficiencia adrenal primaria, ya que el principal responsable es la deficiencia de mineralocorticoide, resultando en una descompensación hemodinámica aguda (hipotensión, taquicardia, hipovolemia, desorientación o alteración de la consciencia asociado a alteraciones electrolíticas) (11). Entre los diversos precipitantes, los más comunes son el estrés emocional, trauma, infarto/hemorragia adrenal, infección gastrointestinal y fiebre $(8,11)$. El diagnóstico de la crisis adrenal debe considerarse en todo paciente con o sin enfermedad adrenal o hipofisiaria diagnosticada previamente. Cursa con fiebre, hipoglicemia, hipotensión, dolor abdominal, náuseas severas, confusión, hiperkalemia e hiponatremia (8). Sin embargo, es más frecuente que ocurra en un paciente con una insuficiencia adrenal establecida, ésta a la vez aumenta su mortalidad $(8,12)$. A la hora de realizar la historia clínica es importante tener en cuenta los diferentes precipitantes de la crisis adrenal, además de historia reciente de suspensión abrupta de corticosteroides a largo plazo, pacientes que habitan en zonas endémicas de tuberculosis, 
metástasis en pacientes con alguna enfermedad oncológica o mala adherencia al tratamiento (12).

La presentación crónica de la insuficiencia adrenal primaria difiere de la insuficiencia adrenal secundaria y terciaria, ya que en esta primera afecta ambas líneas adrenales: glucocorticoide y mineralocorticoide a la vez, mientras que en la secundaria y terciaria solo alteran la línea glucocorticoide, preservando la mineralocorticoide; por consiguiente, la presentación clínica varía (8). Entre las diferentes manifestaciones clínicas de la insuficiencia adrenal primaria, se encuentran las asociadas a insuficiencia mineralocorticoide: hipotensión ortostática, hiponatremia hipovolémica (70-80\%), hiperkalemia (40\%) y antojos salados (64\%). Por otro lado, la deficiencia glucocorticoide se aprecia clínicamente como fatiga (<95\%), pérdida de peso (70-100\%), mialgias (40\%) y también se ha visto implicada con hiponatremia (8). De igual manera, se puede apreciar anemia normocítica normocrómica (10-15\%), hipovolemia, hipercalcemia (6\%) y acidosis metabólica leve (8).

Otro hallazgo muy común que se aprecia en el 90\% de los pacientes es la hiperpigmentación de áreas fotoexpuestas, mucosa bucal, cicatrices, surcos palmares, pezones y zonas expuestas a fricción/presión como por ejemplo rodillas, codos, nudillos $(8,11,12)$. Por otro lado, la secreción de andrógenos adrenales se ve alterada ocasionando clínicamente pérdida de vello púbico y axilar en mujeres (11).

En el anexo 2 se mencionan algunos de los diferentes diagnósticos diferenciales de la insuficiencia adrenal primaria.

\begin{tabular}{|c|c|c|c|}
\hline \multicolumn{2}{|c|}{ Diagnóstico diferencial } & Síntomas y signos clínicos & Hallazgos de Laboratorio \\
\hline $\begin{array}{l}\text { Insuficiencia } \\
\text { secundaria }\end{array}$ & adrenal & $\begin{array}{l}\text {-Fatiga, mialgias, pérdida } \\
\text { de peso, euvolemia, } \\
\text { normotensivo, piel pálida } \\
\text {-Síntomas relacionados } \\
\text { con efecto de masa } \\
\text { pituitaria (cefalea, } \\
\text { alteraciones en los campos } \\
\text { visuales, galactorrea), } \\
\text { síntomas relacionados } \\
\text { con deficiencia de otras } \\
\text { hormonas de la hipófisis } \\
\text { anterior (amenorrea) }\end{array}$ & $\begin{array}{l}\text {-Balance ácido base normal } \\
\text {-Hiponatremia euvolémica } \\
\text {-Normocalemia } \\
\text {-Hipoglicemia } \\
\text {-Calcio normal }\end{array}$ \\
\hline $\begin{array}{l}\text { Insuficiencia } \\
\text { terciaria }\end{array}$ & adrenal & $\begin{array}{l}\text {-Fatiga, mialgias, pérdida } \\
\text { de peso, euvolemia, } \\
\text { normotensivo, piel pálida } \\
\text {-Antecedente de exposición } \\
\text { a glucocorticoides } \\
\text { exógenos } \\
\text { - Paradójicamente con } \\
\text { apariencia Cushingoide } \\
\text { debido a los efectos del } \\
\text { tratamiento esteroideo } \\
\text { recientemente suspendido }\end{array}$ & \\
\hline
\end{tabular}

Anexo 2. Diagnósticos diferenciales de insuficiencia adrenal. SAI (Insuficiencia adrenal secundaria), TAl (Insuficiencia adrenal terciaria) (Martin, Agnieszka). 


\section{DIAGNÓSTICO}

Esta enfermedad debe ser sospechada en todos los pacientes que se presenten agudamente o crónicamente enfermos con los síntomas anteriormente mencionados. La presentación clásica es baja concentración de cortisol ( $<5 \mathrm{ug} / \mathrm{dl}$ ) con una ACTH elevada $(>100 \mathrm{pg} / \mathrm{ml})(6)$. También hay una deficiencia de mineralocorticoides que se presenta como disminución de sodio y elevación de potasio. Puede existir baja aldosterona y renina alta. El diagnóstico se realiza con la prueba corta de cosintropina (test de ACTH o test de Synacthen) (4,7). Los adultos reciben 250 ug intravenoso de tetracosactida (componente de la $\mathrm{ACTH}$ ), luego de la administración se toma el cortisol en sangre inmediatamente, a los 30 y 60 minutos. Concentración de $<500 \mathrm{nmol} / \mathrm{L}$ de cortisol es aceptado como diagnóstico (4). Luego de la confirmación de la insuficiencia suprarrenal, es necesario evaluar el ACTH en plasma para clasificar entre la insuficiencia primaria y secundaria (7).

Algunos datos útiles para el diagnóstico son la hiponatremia, hiperkalemia y/o hipoglicemia (4). Otra prueba útil es la cuantificación al azar de cortisol (7).

\section{TRATAMIENTO}

El manejo de la enfermedad de Addison se basa en el reemplazo de glucocorticoides y en ocasiones, mineralocorticoides de por vida. Hidrocortisona es el medicamento de elección y se da en una dosis de 1520 mg vía oral por día dividido en $2-3$ dosis $(13,14,15)$. Se prefiere que la dosis matutina sea la de mayor cantidad debido a que de esta forma se simula la liberación pulsátil que se ve en el ritmo circadiano normal $(14,15)$. De no ser posible el tratamiento con hidrocortisona, se recomienda prednisona 3-5 mg vía oral por día en 1-2 dosis $(13,14,15)$. No se recomienda el tratamiento con dexametasona debido a que presenta una farmacocinética muy diferente a la fisiología normal. La respuesta al tratamiento se monitoriza mediante parámetros clínicos como el peso, presencia de edemas, presión arterial elevada y presencia de síndrome de Cushing. Cuando los pacientes presentan deficiencia de mineralocorticoides, el medicamento de elección es fludrocortisona $(14,15)$. Se recomienda que las dosis de estos fármacos sean aumentadas al doble o triple cuando los pacientes presentan situaciones de estrés excesivo (cirugías, infecciones, entre otros) (14). Las crisis adrenales deben ser tratadas con $100 \mathrm{mg}$ intravenosos de hidrocortisona, seguidas de una infusión de $200 \mathrm{mg}$ cada 24 horas y fluidoterapia adecuada $(7,14,15)$

\section{PRONÓSTICO}

El tratamiento de la enfermedad de Addison es de por vida y es importante educar a los pacientes acerca de la posibilidad de descompensaciones $(14,15)$. La calidad de vida se ve afectada por una mala adherencia al tratamiento o dosis inadecuadas. La mortalidad y morbilidad de estos pacientes se ve disminuida con el reemplazo de glucocorticoides y mineralocorticoides de forma adecuada $(7,14,15)$.

\section{CONCLUSIÓN}

La enfermedad de Addison o la insuficiencia suprarrenal primaria es una patología que se caracteriza por falla en la síntesis de cortisol en la glándula suprarrenal. Esto genera una serie de síntomas como; malestar general, debilidad, hipotensión, deshidratación, hiperpigmentación, etc. Se presenta con cortisol bajo, una ACTH elevada y el gold standard para el diagnóstico definitivo de esta enfermedad es la prueba corta de cosintropina. El tratamiento se basa en el reemplazo de glucocorticoides y mineralocorticoides de por vida. El fármaco de elección es la Hidrocortisona o prednisona. En pacientes adecuadamente tratados, la enfermedad no afecta la calidad de vida. Sin embargo, la aparición de una crisis suprarrenal sí aumenta la mortalidad. 


\section{REFERENCIAS BIBLIOGRÁFICAS}

1. Betterle C, Presotto F, Furmaniak J. Epidemiology, pathogenesis, and diagnosis of Addison's disease in adults. J Endocrinol Invest. 2019 Dec;42(12):1407-1433. doi: 10.1007/s40618-019-01079-6.

2. Barthel A, Benker G, Berens K, Diederich S, Manfras B, Gruber M, Kanczkowski W, Kline G, Kamvissi-Lorenz V, Hahner S, Beuschlein F, Brennand A, Boehm BO, Torpy DJ, Bornstein SR. An Update on Addison's Disease. Exp Clin Endocrinol Diabetes. 2019 Feb;127(2-03):165-175. doi: 10.1055/ a-0804-2715.

3. Joersjö P, Block L. A Challenging diagnosis that eventually results in a life-threatening condition: Addison's disease and adrenal crisis. BMJ Case Rep. 2019 Dec 29;12(12):e231858. doi: 10.1136/bcr2019-231858.

4. Barthel A, Benker G, Berens K, Diederich S, Manfras B, Gruber M, et al. An update on addison's disease. Exp Clin Endocrinol Diabetes. 2019;127(2-03):165-75. doi:10.1055/a-0804-2715

5. Hellesen A, Bratland E, Husebye ES. Autoimmune Addison's disease - An update on pathogenesis. Annales d'Endocrinologie. 2018;79(3):157-163.doi:10.1016/j.ando.2018.03.008

6. Saverino S, Falorni A. Autoimmune Addison's disease. Best Pract Res Clin Endocrinol Metab. 2020;34(1):101379.doi:10.1016/j.beem.2020.101379

7. Kasper D, Hauser S, Jameson J, Fauci A, Longo D, Loscalzo J. Principios de Medicina Interna (2 T.). 19th ed. Mexico, DF: McGraw-Hill Professional Publishing; 2016

8. Martin-Grace, Julie et al. "Adrenal insufficiency: Physiology, clinical presentation and diagnostic challenges." Clinica chimica acta; international journal of clinical chemistry vol. 505 (2020): 78-91. doi:10.1016/j.cca.2020.01.029

9. Boron W, Boulpaep E, Buccino G, Possenti R, Barret E. Fisiologia medica. 3rd ed. España: Elsevier; 2018

10. Hellesen A, Bratland E, Husebye E. Autoimmune Addison's disease - An update on pathogenesis. Annales d'Endocrinologie. 2018;79(3):157-163.

11. Pazderska, Agnieszka, and Simon Hs Pearce. "Adrenal insufficiency - recognition and management." Clinical medicine (London, England) vol. 17,3 (2017): 258-262. doi:10.7861/ clinmedicine.17-3-258

12. Feeney, $\mathrm{C}$ et al. "Addisonian crisis: assessment and management." British journal of hospital medicine (London, England : 2005) vol. 79,3 (2018): C34-C37. doi:10.12968/hmed.2018.79.3.C34

13. Goubar T, Torpy D, McGrath S, Rushworth R. Prehospital Management of Acute Addison Disease: Audit of Patients Attending a Referral Hospital in a Regional Area. Journal of the Endocrine Society. 2019;3(12):2194-2203.

14. Barthel A, Benker G, Berens K, Diederich S, Manfras B, Gruber M et al. An Update on Addison's Disease. Experimental and Clinical Endocrinology \& Diabetes. 2018;127(02/03):165-175.

15. Bornstein S, Allolio B, Arlt W, Barthel A, Don-Wauchope A, Hammer G et al. Diagnosis and Treatment of Primary Adrenal Insufficiency: An Endocrine Society Clinical Practice Guideline. The Journal of Clinical Endocrinology \& Metabolism. 2016;101(2):364-389. 